\title{
Bourse de recherche Norgine sous l'égide de la Société Française D'endoscopie Digestive. Optimisation de la préparation colique
}

\author{
A. Fima \\ (C) Lavoisier SAS 2016
}

En 2014, Norgine a souhaité favoriser des projets de recherche dans le domaine de la coloscopie et a créé une Bourse de recherche Norgine en partenariat avec la Société Française d'Endoscopie Digestive.

Cette bourse vise à favoriser la recherche et les bonnes pratiques dans le domaine de la coloscopie, et à promouvoir des projets de recherche clinique centrés sur l'éducation des patients, les indicateurs de qualité de la coloscopie et la qualité de la préparation colique.

Cette année, la bourse d'un montant de $15000 €$ sera décernée lors de Vidéo-Digest le vendredi 4 novembre à Paris.
Le jury est composé des membres du conseil d'administration de la SFED : Dr Philippe Bulois (Président du Jury et Président de la SFED), Pr Michel Robaszkiewicz (Secrétaire Général), Pr Juergen Hochberger (Membre du Conseil d'Administration), Pr Thierry Lecomte (Membre du Conseil d'Administration) et des membres désignés par la SFED : Dr Franck Devulder, Dr Thierry Helbert et Dr Hervé Hagège.

Norgine remercie vivement la SFED pour ce partenariat de longue date.

\footnotetext{
A. Fima $(\bowtie)$

Norgine Pharma, 2 rue Jacques Daguerre, F-92565 Rueil

Malmaison, France

e-mail : afima@norgine.com
} 